\title{
Predictors of hyperglycemia after cardiac surgery in nondiabetic patients
}

\author{
Rajesh Garg, MD, ${ }^{\mathrm{a}}$ Anjali Grover, MD, ${ }^{\mathrm{a}}$ Siobhan McGurk, BS, ${ }^{\mathrm{b}}$ and James D. Rawn, MD
}

Objective: Postoperative hyperglycemia is associated with poor clinical outcomes in patients undergoing cardiac surgery. However, some experts consider hyperglycemia to be an epiphenomenon related to acute stress. We investigated whether preoperative patient characteristics can predict hyperglycemia after cardiac surgery in nondiabetic patients.

Methods: This is a retrospective study of nondiabetic patients undergoing cardiac surgery at a single center during the years 2004 to 2009. Hyperglycemia was defined as 2 consecutive blood glucose readings of $150 \mathrm{mg} / \mathrm{dL}$ or greater during the 72 hours after cardiac surgery.

Results: This study included 1453 patients with hyperglycemia and 2205 patients without hyperglycemia. Hyperglycemic patients were older, were more likely to be men, had higher body mass index, were more likely to be hypertensive and hypercholesterolemic, and had lower left ventricular ejection fractions; in addition, a greater proportion had a history of cardiovascular disease and renal failure. Multivariate logistic regression analysis showed age, gender, body mass index, preoperative serum creatinine, left ventricular ejection fraction, previous cardiac surgery, and preoperative cardiogenic shock to be independently associated with hyperglycemia $(P<.05$ for all). Hyperglycemic patients had more intraoperative and postoperative complications.

Conclusions: Preoperative patient characteristics are associated with hyperglycemia after cardiac surgery.

(J Thorac Cardiovasc Surg 2013;145:1083-7)

Postoperative hyperglycemia after cardiac surgery is associated with an increased risk of complications, including deep sternal wound infections, cardiac arrhythmias, and mortality. ${ }^{1-6}$ Furnary and colleagues ${ }^{1,7}$ showed that tight glycemic control during 72 hours after cardiac surgery with target blood glucose levels less than $150 \mathrm{mg} / \mathrm{dL}$ is associated with a decrease in mortality and morbidity in diabetic patients. Similar observations have been reported in nondiabetic patients. ${ }^{3,6}$ Therefore, glycemic control during the 72 hours after cardiac surgery has become a standard of care. However, there are doubts about the validity of this approach because no randomized controlled trials have been conducted. Moreover, some experts argue that acute illness-related hyperglycemia is the result of an increase in stress hormones and proinflammatory cytokines. ${ }^{8-10}$ Therefore, hyperglycemia may be an epiphenomenon, and its treatment may not be necessary. However, a recent study in critically ill patients showed an association between hyperglycemia

\footnotetext{
From the Division of Endocrinology, ${ }^{\mathrm{a}}$ Diabetes and Hypertension, and Division of Cardiac Surgery, ${ }^{\mathrm{b}}$ Brigham and Women's Hospital, Boston, Mass.

Disclosures: Authors have nothing to disclose with regard to commercial support. Received for publication Nov 10, 2011; revisions received April 27, 2012; accepted for publication July 2, 2012; available ahead of print Aug 27, 2012.

Address for reprints: Rajesh Garg, MD, Division of Endocrinology, Diabetes and Hypertension, Brigham and Women's Hospital, 221 Longwood Ave, Boston, MA 02115 (E-mail: rgarg@partners.org). $0022-5223 / \$ 36.00$

Copyright (c) 2013 by The American Association for Thoracic Surgery http://dx.doi.org/10.1016/j.jtcvs.2012.07.089
}

and mortality even after controlling for the severity of illness. ${ }^{11}$ We hypothesized that preoperative patient characteristics are associated with postoperative hyperglycemia, and therefore, postoperative hyperglycemia may not be purely a stress response.

\section{MATERIALS AND METHODS}

This retrospective cohort study included consecutive patients who underwent cardiac surgery at the Brigham and Women's Hospital between September 2004 and July 2009. Patients with a known history of diabetes, preoperative random plasma glucose greater than $200 \mathrm{mg} / \mathrm{dL}$ or taking antidiabetic medications, or hemoglobin $\mathrm{A} 1 \mathrm{c}(\mathrm{HbA1c})$ greater than $6.5 \%$ were excluded a priori. The project was approved by the Partners Health Human Research Committee.

Data were extracted from the Cardiac Surgery Database maintained according to the definitions of the Society of Thoracic Surgeon's data standards, version 2.52. ${ }^{12}$ Extracted data comprised perioperative parameters, including age, gender, race, weight, height, smoking status, history of diabetes mellitus, hypertension, renal failure, type of cardiac surgery, preoperative plasma glucose, preoperative cardiogenic shock, perfusion time during surgery, number of hours in the intensive care unit (ICU), number of hours on ventilator support, need for insulin infusion, postsurgical complications, and mortality. According to our clinical protocol, blood glucose levels are monitored every 1 to 4 hours in all patients undergoing cardiac surgery for 72 hours postoperatively, and any patient with 2 consecutive blood glucose values of $150 \mathrm{mg} / \mathrm{dL}$ or greater is treated with insulin infusion. Therefore, hyperglycemia was defined as 2 consecutive blood glucose values of $150 \mathrm{mg} / \mathrm{dL}$ or greater.

\section{Statistical Methods}

Data were summarized as numbers with percentage, means \pm standard deviation, or median with interquartile ranges as appropriate. Chi-square analyses were used to compare categoric data. Student $t$ test with Levine's homogeneity of variance was used to compare normally distributed 


\section{Abbreviations and Acronyms \\ $\mathrm{HbA} 1 \mathrm{c}=$ hemoglobin A1c \\ ICU $=$ intensive care unit}

variables, and Mann-Whitney $U$ tests were used for non-normally distributed continuous variables. Risk factors for postoperative hyperglycemia were evaluated using forward stepwise logistic regression. Preoperative variables that were statistically different between groups on univariate analyses and those deemed clinically important were included in the model; we excluded variables occurring after our group assignment criteria. A final enter method logistic regression was constructed that included year of surgery to control for changes in our patient comorbidity and clinical practice over time. Interactions were examined but did not substantially improve the final model. Odds ratios are presented per measure unit for continuous variables. Statistical analyses were done using the Statistical Package for the Social Sciences version 13.0 (SPSS Inc, Chicago, Ill).

\section{RESULTS}

Of the 3658 patients included in our study, 2205 remained euglycemic and postoperative hyperglycemia developed in 1453. Differences between the 2 groups are shown in Table 1. Hyperglycemic patients were older, had higher body mass index, and were more likely to be men, hypertensive, and hypercholesterolemic. They were also more likely to have preexisting renal insufficiency, cardiovascular and peripheral vascular disease, and lower left ventricular ejection fraction. A similar proportion of patients underwent coronary artery bypass surgery or other types of cardiac surgery. Hyperglycemic patients required longer extracorporeal perfusion time during surgery. They also required longer ventilation support and ICU stay, and had a higher overall complication rate and mortality compared with the control group.

A logistic regression model was built including the preoperative variables from Table 1 . Perfusion time, time of ventilator support, and ICU stay were not included in the model because these events occurred after assignment to groups. The results of this analysis are shown in Table 2 . Age, gender, body mass index, serum creatinine, left ventricular ejection fraction, previous cardiac surgery, and preoperative cardiogenic shock were independently associated with hyperglycemia (model performance, $-2 L L$ 4894.49; Cox \& Snell $R^{2}, 0.060$; Nagelkerke $R^{2}, 0.082$; C-statistic, $0.64 ; 95 \%$ confidence interval, $0.62-0.65 ; P<.001$ ).

\section{DISCUSSION}

Our study shows that many preoperative patient characteristics are independently associated with postoperative hyperglycemia after cardiac surgery. Postoperative hyperglycemia is likely to be the result of an interaction between preoperative patient characteristics and acute stress. Consistent with previous data, postoperative complications were more frequent in our hyperglycemic patients. Whether these complications were due to the presence of preoperative risk factors or to hyperglycemia itself cannot be determined from this study. Our data suggest that acute illness-related hyperglycemia is not an epiphenomenon caused by acute illness alone.

An association between hyperglycemia and adverse clinical outcomes after cardiac surgery has been known for some time. ${ }^{13}$ After the publication of Furnary and colleagues' data, ${ }^{7}$ treatment of hyperglycemia has generally been accepted as a standard of care in patients undergoing cardiac surgery. However, randomized controlled trial data confirming the effect of glycemic control in these patients are not available. Randomized controlled trials have been conducted in critically ill patients. In one of these trials, $62 \%$ of the enrolled patients had undergone cardiac surgery. ${ }^{14}$ This trial showed remarkable benefits from tight glycemic control. However, subsequent clinical trials in critically ill patients failed to reproduce these results. ${ }^{15,16}$ The contradictory results of the randomized clinical trials along with unclear pathogenesis of hyperglycemia in this setting have created doubts about the validity of tight glycemic control strategy after cardiac surgery. Our study suggests that patients in whom hyperglycemia develops after cardiac surgery are predisposed to develop hyperglycemia. Therefore, contributors to hyperglycemia after cardiac surgery are multifactorial and include preexisting risk factors in addition to the stress of the surgery and other perioperative events. The same factors that predispose patients to develop hyperglycemia may also contribute to their increased morbidity and mortality. It is difficult to determine from this study whether hyperglycemia per se is responsible for an increased rate of complications or whether insulin infusion is useful in decreasing complications. This study was not designed to determine the association between hyperglycemia and complications or the effect of insulin infusion on complications. It is possible that if hyperglycemic patients had not received insulin infusion in our cohort, complication rates would have been higher. Of note, most recent studies suggest that moderate glycemic control in a range of 120 to $180 \mathrm{mg} / \mathrm{dL}$ is desirable after cardiac surgery. ${ }^{17,18}$

One previous study has shown the presence of metabolic syndrome to be related to hyperglycemia during cardiopulmonary bypass surgery. ${ }^{19}$ We did not capture metabolic syndrome as a discrete diagnosis, but we did find some of its components to be more prevalent in the hyperglycemic patients, for example, obesity and dyslipidemia. Another previous study has shown that the use of angiotensinconverting enzyme inhibitors is associated with hyperglycemia after cardiac surgery. ${ }^{20}$ This may be the result of a higher prevalence of hypertension in the hyperglycemic group. We did not study the effect of preoperative medications. Higher preoperative serum creatinine seemed to be 
TABLE 1. Patient characteristics

\begin{tabular}{|c|c|c|c|c|}
\hline & All & Normoglycemic & Hyperglycemic & \\
\hline $\mathbf{N}$ & 3658 & 2205 & 1453 & $\boldsymbol{P} \leq$ \\
\hline \multicolumn{5}{|l|}{ No. $(\%)$ of patients by year of surgery } \\
\hline 2004 & 125 & $33(26)$ & $92(74)$ & \\
\hline 2005 & 757 & $402(53.1)$ & 355 (46.9) & \\
\hline 2006 & 794 & $474(59.7)$ & $320(40.3)$ & \\
\hline 2007 & 715 & 439 (61.4) & $276(38.6)$ & \\
\hline 2008 & 798 & $515(64.6)$ & $283(35.4)$ & \\
\hline 2009 & 469 & $338(66.3)$ & $158(33.7)$ & \\
\hline \multicolumn{5}{|l|}{ Preoperative patient characteristics } \\
\hline Age $(y)$, mean $(S D)$ & $64.6(13.7)$ & $63.3(14.2)$ & $66.7(12.8)$ & .001 \\
\hline Age $\geq 80 y, \%(n)$ & $14.6(536)$ & $13.5(298)$ & $16.4(238)$ & .017 \\
\hline Female $\%(n)$ & $34.5(1263)$ & $36.1(796)$ & $32.1(467)$ & .014 \\
\hline Not Caucasian race $\%(n)$ & $6.6(240)$ & $6.9(153)$ & $6.0(87)$ & .175 \\
\hline BMI kg/m², mean (SD) & $27.4(5.4)$ & $27.2(5.4)$ & $27.7(5.2)$ & .010 \\
\hline Random blood glucose mg/dL, mean (SD) & $120.9(24.3)$ & $120.6(24.0)$ & $121.3(24.8)$ & .413 \\
\hline Smoker \% (n) & $49.4(1806)$ & $48.7(1073)$ & $50.4(733)$ & .295 \\
\hline Family history of CAD \% (n) & $24.6(900)$ & $24.7(544)$ & $24.5(356)$ & .937 \\
\hline Hypercholesterolemia \% (n) & $64.5(2358)$ & $62.2(1372)$ & $67.9(986)$ & .001 \\
\hline Renal failure \% (n) & $4.1(150)$ & $3.4(75)$ & $5.2(75)$ & .010 \\
\hline Serum creatinine $\mathrm{mg} / \mathrm{dL}$, mean $(\mathrm{SD})$ & $1.07(0.55)$ & $1.06(0.60)$ & $1.09(0.48)$ & .123 \\
\hline Hypertension \% (n) & $37.2(1360)$ & $35.8(789)$ & $39.3(571)$ & .020 \\
\hline Cerebrovascular accident $\%(n)$ & $4.9(181)$ & $4.8(105)$ & $5.2(76)$ & .534 \\
\hline Peripheral vascular disease $\%(n)$ & $12.7(465)$ & $11.3(249)$ & $14.9(216)$ & .002 \\
\hline Cerebrovascular disease $\%(n)$ & $11.2(408)$ & $10.8(238)$ & $11.7(170)$ & .412 \\
\hline Myocardial infarction \% (n) & $20.1(736)$ & $18.5(408)$ & $22.6(328)$ & .003 \\
\hline Congestive heart failure $\%(n)$ & $32.4(1187)$ & $30.2(667)$ & $35.8(520)$ & .001 \\
\hline Ejection fraction \%, mean (SD) & $55.3(12.3)$ & $56.4(11.5)$ & $53.7(13.3)$ & .001 \\
\hline Previous cardiac surgery $\%(n)$ & $9.4(344)$ & $8.3(183)$ & $11.1(161)$ & .007 \\
\hline \multicolumn{5}{|l|}{ Operative characteristics } \\
\hline Perfusion time (min) median (IQR) & $117(89-161)$ & $113(87-154)$ & $122(91-171)$ & .001 \\
\hline Crossclamp time (min) median (IQR) & $84(64-114)$ & $84(64-111)$ & $95(64-117)$ & .029 \\
\hline CABG \% (n) & $31.7(1160)$ & $32.3(712)$ & $30.8(448)$ & .364 \\
\hline Valve surgery $\%(n)$ & $45.2(1652)$ & $46.4(1023)$ & $43.3(629)$ & .067 \\
\hline Valve and CABG \% (n) & $16.7(611)$ & $14.4(318)$ & $20.2(294)$ & .001 \\
\hline Other surgery $\%(n)$ & $6.4(233)$ & $6.8(150)$ & $5.7(83)$ & .190 \\
\hline \multicolumn{5}{|l|}{ ICU outcomes and complications } \\
\hline Total ICU stay (h), median (IQR) & $49.0(27-84)$ & $47.0(26-72)$ & $61.0(31-101)$ & .001 \\
\hline Ventilation time $(h)$, median (IQR) & $7.1(4-12)$ & $6.4(4-11)$ & $8.5(5-15)$ & .001 \\
\hline Ventilation $>24$ h $\%$ (n) & $9.1(332)$ & $5.7(125)$ & $14.3(207)$ & .001 \\
\hline Reoperation for bleeding \% (n) & $2.8(104)$ & $1.6(36)$ & $4.7(68)$ & .001 \\
\hline Deep sternal wound infection \% (n) & $0.6(23)$ & $0.5(11)$ & $0.8(12)$ & .316 \\
\hline Leg infection \% (n) & $0.6(22)$ & $0.5(11)$ & $0.7(11)$ & .442 \\
\hline Sepsis \% (n) & $1.8(64)$ & $1.1(25)$ & $2.7(39)$ & .004 \\
\hline Permanent stroke $\%(n)$ & $3.6(132)$ & $3.3(73)$ & $4.0(59)$ & .386 \\
\hline Pulmonary embolism \% (n) & $0.4(16)$ & $0.4(9)$ & $0.5(7)$ & 1.000 \\
\hline Limb ischemia \% (n) & $0.3(12)$ & $0.2(5)$ & $0.5(7)$ & .300 \\
\hline Cardiac arrest \% (n) & $1.4(52)$ & $0.8(17)$ & $2.4(35)$ & .001 \\
\hline \multicolumn{5}{|l|}{ Hospital outcomes } \\
\hline Length of stay (d) median (IQR) & $7.0(5-10)$ & $6.0(5-10)$ & $7.0(5-7)$ & .001 \\
\hline Operative mortality \% (n) & $2.7(100)$ & $2.2(48)$ & $3.6(52)$ & .017 \\
\hline Readmission $\leq 30 \mathrm{~d} \%$ (n) & $10.8(395)$ & $10.6(235)$ & $11.0(160)$ & .373 \\
\hline
\end{tabular}

$S D$, Standard deviation; $B M I$, body mass index; $C A D$, coronary artery disease; $I Q R$, interquartile range; $C A B G$, coronary artery bypass grafting; $I C U$, intensive care unit.

protective of postoperative hyperglycemia, perhaps because insulin is partly metabolized by the kidneys. Therefore, patients with higher serum creatinine levels (lower glomerular filtration rate) may have higher bioavailability of insulin and lower blood glucose levels. However, this relationship was broken in patients with renal failure who tended to 
TABLE 2. Logistic regression analysis

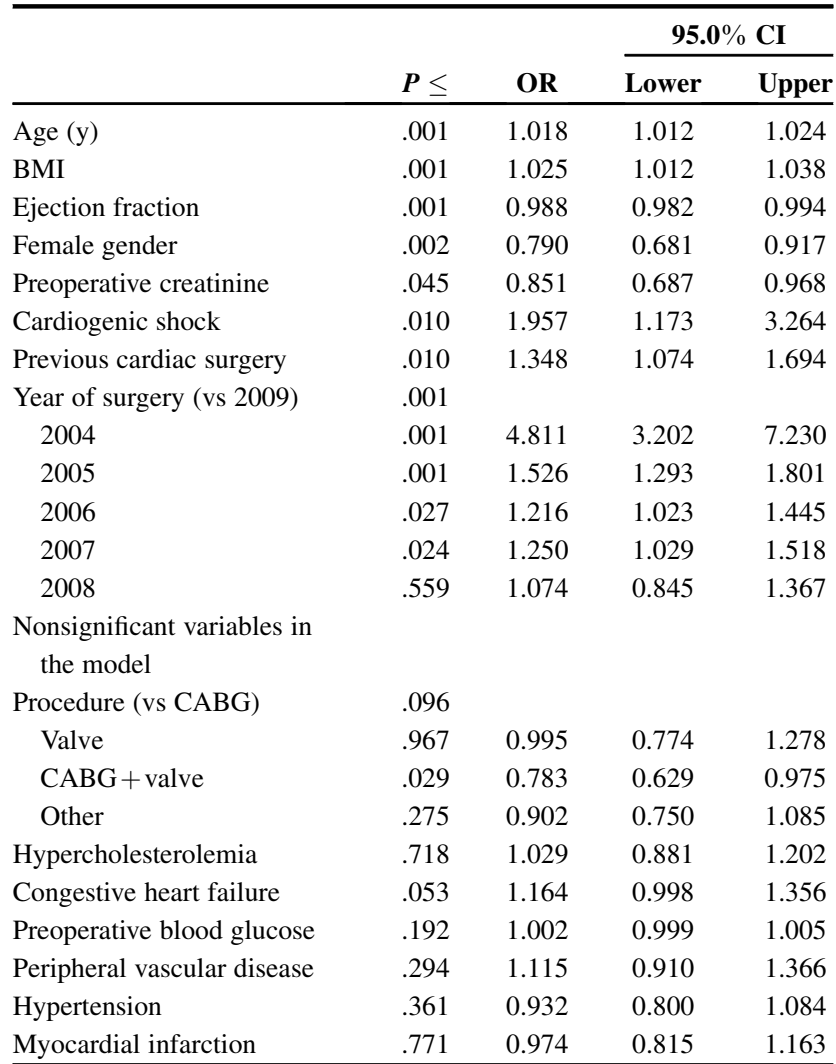

$C I$, Confidence interval; $O R$, odds ratio; $B M I$, body mass index; $C A B G$, coronary artery bypass grafting.

have higher blood glucose levels in our study, possibly secondary to higher stress levels.

\section{Study Limitations}

Our study has the limitations expected of any retrospective data analysis. The year of surgery was included as a surrogate for change in practice, as well as change in nonspecific patient characteristic over time. Of note, we found a significant effect of the year of surgery on the incidence of hyperglycemia. This may be attributable to changes in our clinical practice with regard to hyperglycemia over this time; in particular, patients began receiving insulin infusions during surgery in 2005 and were less likely to be hyperglycemic when arriving in the ICU postoperatively. We think the sudden change from 2004 to 2005 was most likely related to this change in the practice, and changes in subsequent years were probably related to both changes in practice and changes in patient characteristics. Our study includes only 125 patients from 2004. When we repeated our analysis after excluding these patients, the results were essentially unchanged. Another limitation is the possibility of undiagnosed diabetes in our study cohort. Although we took all possible precautions to exclude diabetic patients in this study, some of the patients may still have undiagnosed diabetes because of the high prevalence of diabetes in patients undergoing cardiac surgery. ${ }^{21} \mathrm{~A}$ review of our database revealed that 829 patients $(22.6 \%)$ had an HbA1c measured within 3 months preoperatively. We excluded patients with an $\mathrm{HbAlc}$ greater than $6.5 \%$. Hyperglycemia developed in 728 patients $(88 \%)$ ) with available HbA1c, which was far more than those who did not have an HbAlc. Because HbAlc testing is not routine at Brigham and Women's Hospital, these results likely represent a testing bias where $\mathrm{HbAlc}$ is ordered for patients with higher risk of diabetes. Therefore, it is unlikely that undiagnosed diabetes among patients with no available HbA1c was a major determinant of hyperglycemia. We believe our study cohort is representative of the nondiabetic cardiac surgery patient population.

The results support our hypothesis that preoperative patient characteristics are associated with postoperative hyperglycemia in patients undergoing cardiac surgery. However, the overall predictive value of the regression model is rather weak, suggesting that other factors, for example, severity of sickness, also may play an important role in the occurrence of hyperglycemia. Whether these finding are applicable to other acute illnesses will need further investigation.

\section{CONCLUSIONS}

Preoperative patient characteristics are associated with postoperative hyperglycemia after cardiac surgery. Therefore, postoperative hyperglycemia is likely to be a marker of long-standing abnormalities in these patients, as well as a response to acute illness.

\section{References}

1. Furnary AP, Wu Y, Bookin SO. Effect of hyperglycemia and continuous intravenous insulin infusions on outcomes of cardiac surgical procedures: the Portland Diabetic Project. Endocr Pract. 2004;10(Suppl 2):21-33.

2. Falcao G, Ulate K, Kouzekanani K, Bielefeld MR, Morales JM, Rotta AT. Impact of postoperative hyperglycemia following surgical repair of congenital cardiac defects. Pediatr Cardiol. 2008;29:628-36.

3. Doenst T, Wijeysundera D, Karkouti K, et al. Hyperglycemia during cardiopulmonary bypass is an independent risk factor for mortality in patients undergoing cardiac surgery. J Thorac Cardiovasc Surg. 2005;130:1144.

4. Furnary AP, Wu Y. Clinical effects of hyperglycemia in the cardiac surgery population: the Portland Diabetic Project. Endocr Pract. 2006;12(Suppl 3):22-6.

5. Bucerius J, Gummert JF, Walther T, et al. Impact of diabetes mellitus on cardiac surgery outcome. Thorac Cardiovasc Surg. 2003;51:11-6.

6. Estrada CA, Young JA, Nifong LW, Chitwood WR Jr. Outcomes and perioperative hyperglycemia in patients with or without diabetes mellitus undergoing coronary artery bypass grafting. Ann Thorac Surg. 2003;75:1392-9.

7. Furnary AP, Gao G, Grunkemeier GL, et al. Continuous insulin infusion reduces mortality in patients with diabetes undergoing coronary artery bypass grafting. $J$ Thorac Cardiovasc Surg. 2003;125:1007-21.

8. McCowen KC, Malhotra A, Bistrian BR. Stress-induced hyperglycemia. Crit Care Clin. 2001;17:107-24.

9. Tracey F, Crawford VL, Lawson JT, Buchanan KD, Stout RW. Hyperglycaemia and mortality from acute stroke. Q J Med. 1993;86:439-46.

10. Dungan KM, Braithwaite SS, Preiser JC. Stress hyperglycaemia. Lancet. 2009; 373:1798-807.

11. Falciglia M, Freyberg RW, Almenoff PL, D’Alessio DA, Render ML. Hyperglycemia-related mortality in critically ill patients varies with admission diagnosis. Crit Care Med. 2009;37:3001-9. 
12. STS Adult Cardiac Database. Available at: http://www.ctsnet.org/file/ 4AdultCVDataSpecs252_ForGeneralDistribution.pdf. Accessed April 26, 2012.

13. Golden SH, Peart-Vigilance C, Kao WH, Brancati FL. Perioperative glycemic control and the risk of infectious complications in a cohort of adults with diabetes. Diabetes Care. 1999;22:1408-14.

14. van den Berghe G, Wouters P, Weekers F, et al. Intensive insulin therapy in the surgical intensive care unit. N Engl J Med. 2001;345:1359-67.

15. Van den Berghe G, Wilmer A, Hermans G, et al. Intensive insulin therapy in the medical ICU. N Engl J Med. 2006;354:449-61.

16. Finfer S, Chittock DR, Su SY, et al. Intensive versus conventional glucose control in critically ill patients. $N$ Engl J Med. 2009;360:1283-97.

17. Bhamidipati CM, LaPar DJ, Stukenborg GJ, et al. Superiority of moderate control of hyperglycemia to tight control in patients undergoing coronary artery bypass grafting. J Thorac Cardiovasc Surg. 2011;141:543-51.
18. Desai SP, Henry LL, Holmes SD, et al. Strict versus liberal target range for perioperative glucose in patients undergoing coronary artery bypass grafting: a prospective randomized controlled trial. J Thorac Cardiovasc Surg. 2012;143 318-25.

19. Donatelli F, Cavagna P, Di Dedda G, et al. Correlation between pre-operative metabolic syndrome and persistent blood glucose elevation during cardiac surgery in non-diabetic patients. Acta Anaesthesiol Scand. 2008;52:1103-10.

20. Prasad AA, Kline SM, Schuler HG, Sukernik MR. Clinical and laboratory correlates of excessive and persistent blood glucose elevation during cardiac surgery in nondiabetic patients: a retrospective study. J Cardiothorac Vasc Anesth. 2007;21: 843-6.

21. Abdelmalak B, Abdelmalak JB, Knittel J, et al. The prevalence of undiagnosed diabetes in non-cardiac surgery patients, an observational study. Can J Anaesth. 2010;57:1058-64. 